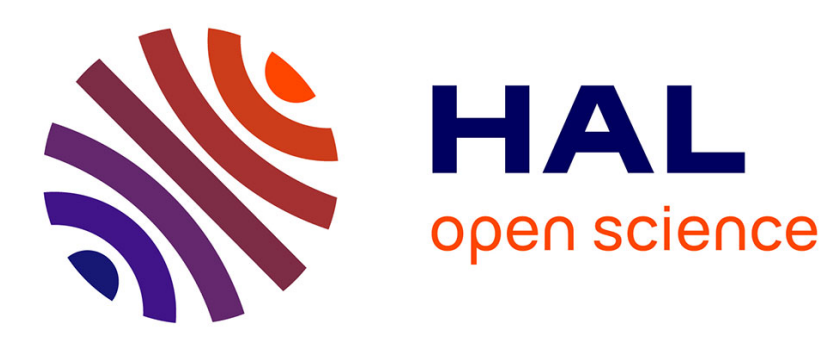

\title{
Monoclinic Al13Fe4 approximant phase: a link between icosahedral and decagonal phases
}

\author{
J.-N. Barbier, N. Tamura, J.-L. Verger-Gaugry
}

\section{To cite this version:}

J.-N. Barbier, N. Tamura, J.-L. Verger-Gaugry. Monoclinic Al13Fe4 approximant phase: a link between icosahedral and decagonal phases. Journal of Non-Crystalline Solids, 1993, 153-154, pp.126-131. 10.1016/0022-3093(93)90328-U . hal-03133806

\section{HAL Id: hal-03133806 https://hal.science/hal-03133806}

Submitted on 15 Feb 2021

HAL is a multi-disciplinary open access archive for the deposit and dissemination of scientific research documents, whether they are published or not. The documents may come from teaching and research institutions in France or abroad, or from public or private research centers.
L'archive ouverte pluridisciplinaire HAL, est destinée au dépôt et à la diffusion de documents scientifiques de niveau recherche, publiés ou non, émanant des établissements d'enseignement et de recherche français ou étrangers, des laboratoires publics ou privés. 


\title{
Monoclinic $\mathrm{Al}_{13} \mathrm{Fe}_{4}$ approximant phase: a link between icosahedral and decagonal phases
}

\author{
J.-N. Barbier, N. Tamura and J.-L. Verger-Gaugry \\ LTPCM/ENSEEG/INPG (CNRS UA 29), BP 75, Domaine Universitaire, 38402 Saint \\ Martin d'Hères, France
}

\begin{abstract}
It is shown that the approximant monoclinic crystal $\mathrm{Al}_{13} \mathrm{Fe}_{4}$, known as a $\left(\mathrm{C}_{5}\right)$ decagonal-approximant crystal for $\mathrm{d}-\mathrm{AlFe}$, is also a valuable approximant for the icosahedral quasicrystalline phase i$\mathrm{AlFeCu}$. The icosahedral local order, in a half-plane around $\mathrm{Fe}(5)$ sites, is similar, up to the sixth neighbor, to the one around the origin or around the body center site (empty sites) in the cubic $(\mathrm{Pm} \overline{3}) \alpha$-AlMnSi phase, with a greater chemical disorder and a stronger tendency to geometric frustration as indicated by more empty sites in the icosahedral shells. This similarity gives some information about possible icosahedral local order in the quasicrystalline phase $\mathrm{i}-\mathrm{AlFeCu}$, and the possible atomic surfaces in a 6D Euclidean hyperspace approach.
\end{abstract}




\section{Introduction}

One key for the determination of the structure of a quasicrystalline phase is the study of its crystalline approximants [1]. When the icosahedral symmetry groups are concerned, we consider crystal approximants, containing atomic configurations exhibiting nearly icosahedral symmetry [1]. When non-crystallographic cyclic or dihedral groups are concerned, we analyze crystals containing atomic clusters that are almost invariant under the action of such groups. We have called these 'G-approximant crystals' in ref. [1], where $\mathrm{G}$ is the non-crystallographic point group. We reserve this term for cases where the symmetry (G) is propagating far from some centers (empty or occupied) in the crystal as in $\alpha$-AlMnSi [2], or $\mathrm{c}-\mathrm{Ti}_{2} \mathrm{Ni}$ [3]. Owing to the great variety of possible atomic clusters, crystals can be simultaneously approximants for several non-crystallographic symmetry point groups, in the sense of ref. [1]. However, apart from structure determination studies of crystals by X-rays, the literature does not, to our knowledge, contain reports on noncrystallographic symmetry type long-range local order. We have reanalyzed many crystals by writing software to calculate the bond orientational coefficients deduced from spherical harmonics to analyze local symmetries $[4,5]$.

This type of investigation is relevant for several reasons. First, it allows us to predict the orientation relationships between the G-approximant crystal and its (G-) quasicrystalline phase when they coexist, since we know that the local order is almost identical in both cases, up to small deformations. Second, when several noncrystallographic point groups are concerned, it enables us to predict the coexistence of several quasicrystalline phases, together with their mutual orientations with the approximant crystal. Third, when there are group/subgroup relations, like $\mathrm{C}_{5} \rightarrow \mathrm{m} \overline{3} \overline{5}$, it allows us to envisage the phase transition between the quasicrystalline phases and the associated approximant crystal in terms of the invariance of their common symmetry axes.

In the present work, we study the 'double approximant' character of the monoclinic crystal $\mathrm{Al}_{13} \mathrm{Fe}_{4}$, for the symmetry groups $\mathrm{C}_{5}$ and $\mathrm{m} \overline{3} \overline{5}$, showing that it is an approximant for the decago- 


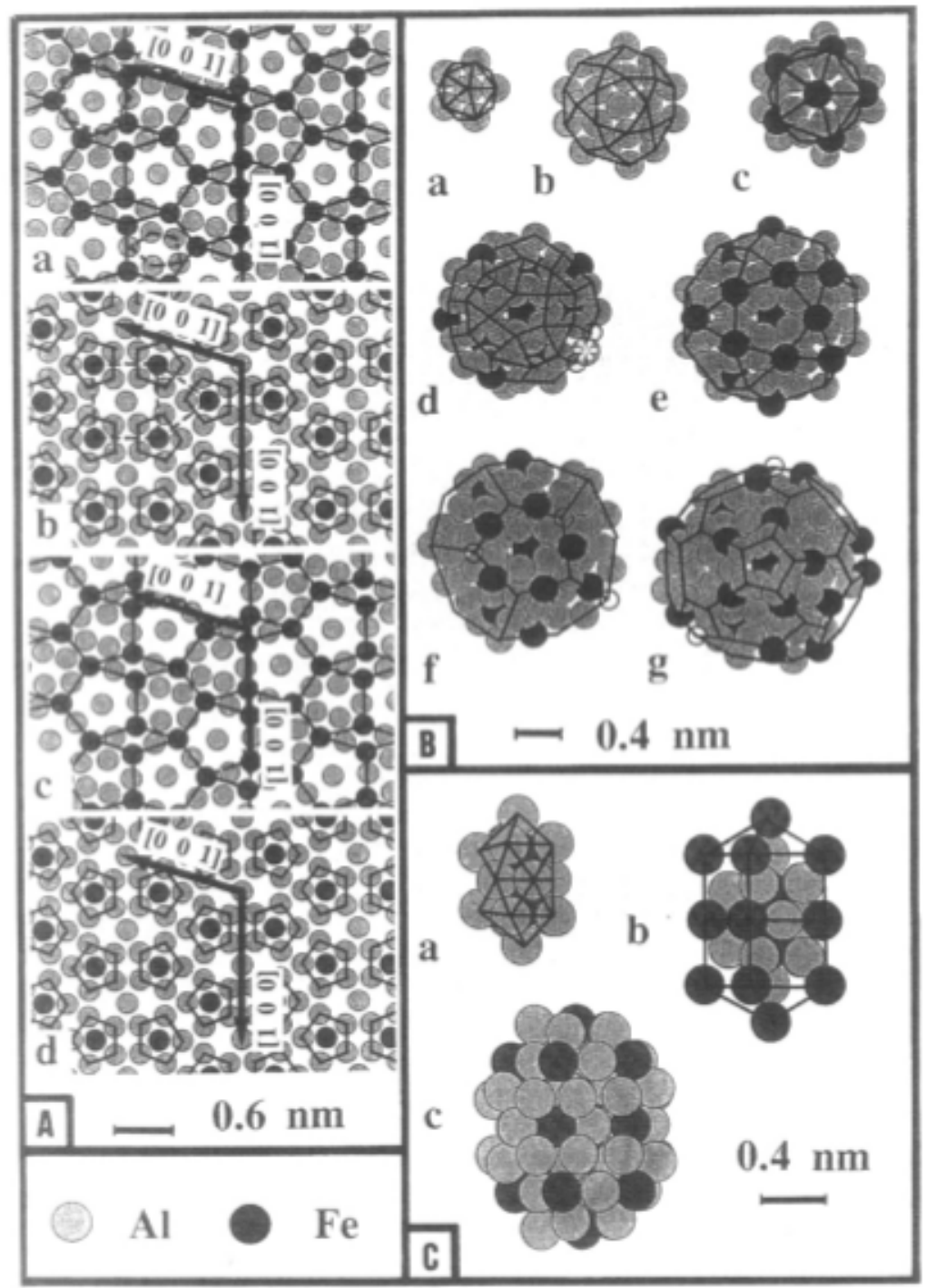

Fig. 1. (A) Layered structure of the approximant crystal $\mathrm{Al}_{13} \mathrm{Fe}_{4}$, perpendicular to the $b$-axis: (a) at $y=0$, (b) at $y=1 / 4$, (c) at $y=1 / 2$, (d) at $y=3 / 4$. Each layer is a decorated 2D periodic approximant tiling of a $2 \mathrm{D}$ Penrose tiling and can be decomposed in flat and thin rhombuses. Here, we use a more appropriate decomposition with pentagons and flat rhombuses for (a) and (c), which fits better the atom positions, and with pentagons, rectangles and isoceles triangles for (b) and (d). The Fe(5) atoms are the Fe atoms represented in (b) and (d). (B) Icosahedral local order present in a half-space around $\mathrm{Fe}(5)$ atoms, limited by the mirror plane perpendicular to the $b$-axis at $y=1 / 4$ (or 3/4). The successive pseudoicosahedral (half-) shells of atomic sites are the following: (a) small Al icosahedron, (b) Al icosidodecahedron, (c) Fe icosahedron, (d) Al, Fe hexecontahedron, (e) Fe, Al icosidodecahedron, (f) incomplete $\mathrm{Al}$ dodecahedron, $(\mathrm{g}) \mathrm{Al}$, Fe pentakisdodecahedron. Open circles designate missing atoms in an icosahedral shell, the white star stands for an $\mathrm{Al}$ atom out of its icosahedral position. (C) Mackay pentagonal prism: decagonal cluster formed by two interpenetrating Mackay icosahedra centered at the mid-edge between two successive Fe(5) atoms in symmetric positions with respect to the mirror plane normal to the $b$-axis at $y=0$, or $1 / 2$. 
nal phase, d-AlFe (and d-AlCuCo by extension), and the icosahedral phase, $\mathrm{i}-\mathrm{AlFeCu}$.

\section{Structure of the $\theta$-phase $\mathbf{A l}_{13} \mathrm{Fe}_{4}$}

This monoclinic phase is isostructural with $\mathrm{Al}_{13} \mathrm{Co}_{4}$, has $\mathrm{C} 2 / \mathrm{m}$ as its space group and the following lattice parameters: $a=1.55 \mathrm{~nm}, b=$ $0.81 \mathrm{~nm}, c=1.25 \mathrm{~nm}, \beta=107.7^{\circ}$ [6]. With a small amount of $\mathrm{Cu}$, it coexists with, and is a decomposition product of the icosahedral phase $\mathrm{i}-\mathrm{AlFeCu}$ [7]. It was also used to model the decagonal phases d-AlFe, d-AlCuCo and d-AlCoNi $[8,9]$, because of the almost quasiperiodic distribution of the atoms in the layers perpendicular to the $b$-axis (see section 2.1.), and is considered as the prototype of approximate crystals for the decagonal symmetry [10]. The pseudo-tenfold axis is parallel to the $b$-axis.

\subsection{Layered structure of the $\theta$-phase and $2 D C_{5^{-}}$ approximant tilings}

The $\theta$-phase is composed of four layers stacked perpendicular to the direction of the $b$-axis. The layer at $y=0$ (fig. $1 \mathrm{~A}(\mathrm{a})$ ) (with $0 \leq y \leq 1$ measuring a fraction of the lattice parameter $b$ in the $b$-direction as usual) contains large $\mathrm{Fe}$ pentagons either with an inner $\mathrm{Al}$ pentagon of similar size and a central $\mathrm{A}$ atom, or an inner distorted $\mathrm{Al}$ pentagon with no atom at the center. The inner $\mathrm{Al}$ pentagon is rotated by $\pi / 5$ with respect to the $\mathrm{Fe}$ pentagon. $\mathrm{Fe}$ atoms are on the vertices of a decagonal approximant tiling constituted by regular pentagons and flat rhombuses (edge length $=$ $0.48 \mathrm{~nm}$ ). Small Al pentagons with the same orientations as these Fe pentagons lie on the next layer at $y=1 / 4$ (fig. $1 \mathrm{~A}(\mathrm{~b})$ ). There may be an $\mathrm{Fe}$ atom, or no atom, at the center of these $\mathrm{Al}$ pentagons. $\mathrm{Fe}$ atoms are on the vertices of a decagonal 2D approximant tiling constituted by isoceles triangles and rectangles with edge lengths 0.67 and $0.77 \mathrm{~nm}$. The $\mathrm{Al}$ atoms are on the vertices of a approximant tiling constituted by the same elementary tiles: isoceles triangles, rectangles and also pentagons, but deflated by $\tau^{2}$ with respect to those in the Fe tiling. The length 0.77 $\mathrm{nm}$ is $\tau$ times ( $\tau$ is the golden mean) larger than the edge length of the Fe pentagons of the previous layer at $y=0$. The layer at $y=1 / 2$ (fig. $1 \mathrm{~A}(\mathrm{c}))$ is obtained from the first layer at $y=0$ by the translation $a / 2+b / 2$. We can also get it from the first layer (at $y=0$ ) by a rotation of $\pi$ around the $b$-axis and a translation of $b / 2$. The layer at $y=3 / 4$ is identical to the layer at $y=$ $1 / 4$ (fig. $1 \mathrm{~A}(\mathrm{~d})$ ).

Therefore, we obtain two families of similarly oriented pentagons, up to homothety, i.e. two 'orientational families' [2,3], which are related to each other by a mirror plane containing the $b$-axis and either the $a$-axis or the $c$-axis. These two orientations restore the pseudodecagonal symmetry of the layers, and therefore, of the crystal itself. The layer thickness is equal to $(b / 4=) t=$ $0.2 \mathrm{~nm}$ and corresponds to the mid-height of a small icosahedron. For comparison, $8 t=1.62 \mathrm{~nm}$ is the stacking period of the decagonal phase $\mathrm{d}$-AlFe [11], and also of d-AIPd [12], and $4 t=0.80$ $\mathrm{nm}$ is the one for d-AlCuCo [13].

The superposition of small and large pentagons with similar orientation suggests the existence in the structure of parts of double icosahedra. The center of these partial icosahedra are the $\mathrm{Fe}$ sites, referred to as $\mathrm{Fe}(5)$ atoms by Black [6].

\subsection{Icosahedral and decagonal environments around $\mathrm{Fe}(5)$ sites}

These $\mathrm{Fe}(5)$ atoms are located on the layers $y=1 / 4$ and $y=3 / 4$ (fig. 1). They are surrounded on one side (in one of the two half-spaces limited by the mirror plane normal to the $b$-axis which contains this atom) by a sequence of pseudoicosahedral (half-) polyhedra of atomic sites. The layers, represented in fig. 1B are the following ones: (a) an $\mathrm{Al}$ icosahedron (ico1), (b) an $\mathrm{Al}$ icosidodecahedron (icosi1), (c) an $\mathrm{Fe}$ icosahedron (ico2), (d) an $\mathrm{Al}$ hexecontahedron (hexe1) with a small amount of $\mathrm{Fe}$, (e) an $\mathrm{Fe}, \mathrm{Al}$ icosidodecahedron (icosi2), and (f) a Fe, Al pentakisdodecahedron (pentakis1) fig. $1 \mathrm{~B}(\mathrm{~g})$. Parts of a dodecahedron (dodeca1), fig. 1B(f) and of an icosahedron (ico3), visible in fig. $1 \mathrm{~B}(\mathrm{~d})$ exist between hexe1 and pentakis1. The sequence ico1-icosi1-ico2 
corresponds to the Mackay icosahedron. Larger pseudoicosahedral atomic shells, which are not reported here, can be guessed outside this cluster: an incomplete hexecontahedron (hexe2), an incomplete trisicosahedron (trisico1) and a fourth icosahedron (ico4). The global and layer-by-layer deformations of this cluster are reported in table 1 where we have applied a suitable least-squares method described in ref. [5] to find the correct symmetry type of the different shells in the cluster. The spreading of the icosahedral symmetry in this half-space is relatively important since all the crystalline orbits of the structure are described by the set 'ico1-icosi1-ico2-hexe1', which is mostly the Mackay icosahedron. We see that the interpenetration of (half-)pseudoicosahedral units occurs in the structure of $\mathrm{Al}_{13} \mathrm{Fe}_{4}$.

This local environment is very similar to the one in the cubic $\alpha$-AIMnSi phase (space group $\operatorname{Pm} \overline{3}, a=1.268 \mathrm{~nm}[2,14])$, where atoms are located around the origin (empty site) on the vertices of the following concentric pseudoicosahedral polyhedra: $\mathrm{Al}$ icosahedron (ico1), $\mathrm{Al}$ icosidodecahedron (icosi1), $\mathrm{Mn}$ icosahedron (ico2), $\mathrm{Al}$ hexecontahedron (hexe1), Al icosahedron (ico3), $\mathrm{Mn}, \mathrm{Al}$ icosidodecahedron (icosi2), $\mathrm{Al}, \mathrm{Mn}$ pentakisdodecahedron (pentakis1), hexecontahedron (hexe2), trisicosahedron (trisico1), $\mathrm{Al}$ icosahedron (ico4). The icosahedral local environment around the (empty) body center site is similar but the third icosahedron is replace d by a partial dodeca. hedron.

Let us say in a natural way that, when all the vertices of an icosahedral polyhedron constituting a layer in a cluster are not occupied by the same type of atom, 'a chemical disorder for the icosahedral symmetry' relatively to this layer occurs. Now, we see from the above description that the chemical disorder of the cluster in $\mathrm{Al}_{13} \mathrm{Fe}_{4}$ begins with the fourth layer, while it starts with the sixth layer in the icosahedral cluster (centered at the origin) in the $\alpha$-AIMnSi phase. Similarly, the presence of more incomplete pseudoicosahedral layers in the (half-) cluster in $\mathrm{Al}_{13} \mathrm{Fe}_{4}$ than in $\alpha$-AlMnSi reveals the higher degree of geometric frustration [15] induced by $\mathrm{Fe}$ atoms than by $\mathrm{Mn}$ atoms. A reason for this is also that the cluster center is a $\mathrm{Fe}(5)$ atom in one case, whereas it is not occupied in the second case, distorting the first $\mathrm{Al}$ icosahedron and therefore the above layers. However, we observe the same succession of layers in the pseudoicosahedral clusters in both phases: ico1-icosi1-ico2-hexe1-ico3-icosi2pentakis1.

In the other half-space around $\mathrm{Fe}(5)$ sites, atoms are not on the vertices of icosahedral polyhedra, apart from the first neighbors which are almost in the icosahedral positions obtained by

Table 1

Characterization of the degree of perfection of the icosahedral symmetry in the (half-)pseudoicosahedral cluster centered at a Fe(5) atomic site, up to the second icosidodecahedron. The angular deviations and the atomic displacements for the atoms in each layer are measured between the cluster itself and an optimally positioned perfectly icosahedral cluster [2,5]. Comparison is made with the pseudoicosahedral cluster centered at the origin in the $\alpha$-AIMnSi phase, which is one order of magnitude less distorted

\begin{tabular}{|c|c|c|c|c|c|c|}
\hline \multirow[t]{2}{*}{ Layer } & \multirow[t]{2}{*}{ Atom type } & \multirow[t]{2}{*}{$\begin{array}{l}\text { Average radius } \\
\text { [nm] }\end{array}$} & \multirow[t]{2}{*}{ Occupancy rate } & \multirow[t]{2}{*}{$\begin{array}{l}\text { Max./mean } \\
\text { angular deviation } \\
\text { [deg] }\end{array}$} & \multicolumn{2}{|c|}{$\begin{array}{l}\text { Max. } / \text { mean } \\
\text { atomic displacement } \\
{\left[10^{-2} \mathrm{~nm}\right]}\end{array}$} \\
\hline & & & & & $\theta$ & $\alpha$ \\
\hline icol & $\mathrm{Al}$ & 0.25 & 1 & $26.6 / 14.0$ & $11.8 / 6.7$ & $0.15 / 0.15$ \\
\hline icosil & Al & 0.48 & 1 & $6.8 / 2.7$ & $7.7 / 3.4$ & $0.86 / 0.80$ \\
\hline ico2 & $\mathrm{Fe}$ & 0.47 & 1 & $2.8 / 2.3$ & $2.3 / 2.0$ & $0.17 / 0.17$ \\
\hline hexel & $\mathrm{Al}, \mathrm{Fe}$ & 0.67 & 0.93 & $15.0 / 7.0$ & $18.1 / 6.9$ & $3.34 / 1.48$ \\
\hline (ico3) & - & - & - & - & - & $2.08 / 2.08$ \\
\hline dodecal & Al & 0.77 & 0.7 & - & - & - \\
\hline icosi2 & $\mathrm{Al}, \mathrm{Fe}$ & 0.77 & 1 & $15.1 / 6.1$ & $20.6 / 5.7$ & $2.21 / 1.61$ \\
\hline
\end{tabular}


completing by mirror symmetry the small icosahedron lying in the other half-space.

\subsection{Large decagonal clusters}

Each $\mathrm{Fe}(5)$ site is associated with another one which is symmetric by a mirror plane perpendicular to the $b$-axis (plane at $y=0$ or $y=1 / 2$ ). The association of the local icosahedral environments of the two sites form a big cluster having the pentagonal symmetry (fig. 1C). The association of two small partial Al icosahedra (fig. 1C(a)) together with two $\mathrm{Fe}$ icosahedra forms a pentagonal prism with inner edges decorated by $\mathrm{Al}$ atoms, and three additional atoms belonging to the mirror plane (fig. $1 \mathrm{C}(\mathrm{b})$ ). When the remaining $\mathrm{Al}$ atoms of the icosidodecahedra are added, we obtain a 76-atom cluster, centered at the mirror plane $(y=0$ or $y=1 / 2)$. We will refer to this cluster as the Mackay pentagonal prism because it is formed by joining two incomplete Mackay icosahedra which are symmetric with respect to the mirror plane, perpendicular to the fivefold axis. The $\theta$-phase is formed by the connection and the interpenetration of such Mackay pentagonal prisms. A single Mackay pentagonal prism is actually sufficient to generate the entire crystal from the space group generators since it contains atoms of all the orbits. These prisms have two different orientations connected by a mirror plane which contains the $b$-axis and either the $a$-axis or the $c$-axis, as in the decorated layers with the pentagons (section 2.1.).

\section{Comments}

The orientation relationships between the approximant crystal $\mathrm{Al}_{13} \mathrm{Fe}_{4}$ and $\mathrm{i}$ - $\mathrm{AlFeCu}$ (resp. $\mathrm{d}-\mathrm{AlFe}$ ), deduced from the above study of local order, are the following and are coherent with those reported in literature:
i [17]: i5 $\|[010]$
i2 $\|[100]$ and [001],
d [11]: $d 10 \|$ i5 $\|[010] \quad d 2\|[100]$ and [001].

The lower degree of perfection of the pseudoicosahedral clusters in $\mathrm{Al}_{13} \mathrm{Fe}_{4}$, than in $\alpha$ - $\mathrm{Al}$ $\mathrm{MnSi}$, as it appears from table 1 and ref. [2], indicates that icosahedral local order is slighty different in the phases $\mathrm{i}-\mathrm{AlFeCu}$ and $\mathrm{i}-\mathrm{AlMnSi}$, but with basically the same sequence of shells in the clusters. At least, clusters are centered on some $\mathrm{Fe}$ atoms in the first case and empty sites in the second case. Within the framework of the cut method from the 6D Euclidean space, this provides a large atomic surface, with $\mathrm{Al}$ outside and $\mathrm{Fe}$ inside, at the nodes of the $6 \mathrm{D}$ cubic primitive lattice, and small $\mathrm{Al}, \mathrm{Cu}$ atomic surfaces on midedge and body center positions. Since $\mathrm{i}-\mathrm{AlFeCu}$ is a fcc icosahedral phase, there must exist a second kind of icosahedral local environment. By comparison with other approximant crystals coexisting with $\mathrm{i}-\mathrm{AlMnSi}[2]$, we suggest that the second kind of cluster may be constituted by a small Al, $\mathrm{Fe}$ icosahedron, an $\mathrm{Al}$ dodecahedron, an $\mathrm{Al}$ icosahedron, and an $\mathrm{Al}$ icosidodecahedron. This gives a second atomic surface (when referred to the origin) which is constituted by a large $\mathrm{Al}$ atomic surface at the origin and at the body center site and a small $\mathrm{Al}, \mathrm{Fe}$ one at the edge centers. Chemical ordering about $\mathrm{Cu}$ atoms may occur. This hyperspace description is partially coherent with direct analyzis of the i-AlFeCu phase [16].

From the point of view of rapid solidification from the melt, seeds constituted by $\mathrm{Fe}$ atoms surrounded by $12 \mathrm{Al}$ atoms in the liquid can give rise either to full icosahedral clusters, as described above, and to the i-phase by aggregation and growth, or, by the introduction of a mirror plane, to Mackay pentagonal prisms and the formation of the d-phase [17]. The bifurcation between both regimes is dictated by energetic considerations on the growing clusters, and especially when a mirror plane is introduced, possibly by twinning.

\section{Conclusion}

We have demonstrated that the $\theta$-phase $\mathrm{Al}_{13} \mathrm{Fe}_{4}$, well-known as a decagonal-approximant crystal, is also a valuable approximant for the $\mathrm{i}$-AIFeCu phase. The pseudo-tenfold symmetry is obtained by mirror planes containing the pseudo-fivefold directions. However, this crystal does not seem to be sufficient to predict all the 
atomic surfaces in the $\mathrm{Fm} \overline{3} \overline{5} \mathrm{i}-\mathrm{AlFeCu}$ phase, since it seems to contain only one type of pseudoicosahedral cluster; a second type is expected for symmetry reasons.

\section{References}

[1] J.-L. Verger-Gaugry, J. Phys. I (Paris) 1 (1991) 1303.

[2] N. Tamura and J.-L. Verger-Gaugry, to be submitted to Philos. Mag. A, to be published;

J.-L. Verger-Gaugry, Int. Symp. on the Physics and Chemistry of Finite Systems: From Clusters to Crystals, Richmond, Virginia, USA (Kluwer Academic, Boston, London, 1991).

[3] N. Tamura, A. François, A. Loiseau and J.-L. VergerGaugry, submitted to Philos. Mag. B, to be published.

[4] P.J. Steinhardt, D.R. Nelson and M. Ronchetti, Phys. Rev. B28 (1983) 784.

[5] J.-L. Verger-Gaugry, N. Tamura and J.-N. Barbier, to be submitted to J. Phys. I (Paris).

[6] P.J. Black, Acta Crystallogr. 8 (1955) 43.
[7] C. Janot, M. Audier, M. de Boissieu and J.-M. Dubois, Europhys. Lett. 14 (1991) 355.

[8] A. Yamamoto and K.N. Ishihara, Acta Crystallogr. A44 (1988) 707.

[9] A. Yamamoto, K. Kato, T. Shibuya and S. Takeuchi, Phys. Rev. Lett. 65 (1990) 1603.

[10] A. Janner, Acta Crystallogr. A47 (1991) 577.

[11] K.K. Fung, C.Y. Yang, Y.Q. Zhou, W.S. Zhan and B.G. Shen, Phys. Rev. Lett. 56 (1986) 2060.

[12] S. Idziak, P.A. Heiney and P.A. Bancel, Mater. Sci. Forum 22-24 (1987) 353.

[13] L.X. He, Y.K. Wu and K.H. Kuo, J. Mater. Sci. 7 (1988) 1284

[14] M. Cooper and K. Robinson, Acta Crystallogr. 6 (1966) 614.

[15] J.-F. Sadoc and R. Mosseri, in: Aperiodicity and Order, Extended Icosahedral Structures, Vol. 3, eds M.V. Jaric and D. Gratias (Academic Press, 1989) p. 163.

[16] M. Cornier-Quiquandon, A. Quivy, S. Lefebvre, E. Elkaim, G. Heger, A. Katz, and D. Gratias, Phys. Rev. B44 (1991) 2071.

[17] Y.F. Cheng, M.J. Hui and F.H. Li, Philos. Mag. Lett. 64 (1991) 129. 